\section{René Cardinal \\ Pierre Pagé \\ Mohammad Shenasa \\ Gérard Faugère \\ Pierre Savard}

\title{
Cartographie de l'activation cardiaque et troubles du rythme
}

L'enregistrement d'électrogrammes à l'aide d'électrodes multiples permet de tracer une carte de l'activation épicardique et endocardique au cours des tachycardies ventriculaires. La localisation des zones arythmogènes permet de guider l'acte chirurgical antiarythmique.

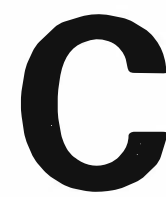
ertains troubles du rythme cardiaque se traduisent par une accélération excessive de la fréquence de battement des ventricules et constituent une menace sérieuse au bien-être et à la survie du malade. Ces tachycardies peuvent être dues à la présence d'une voie de conduction accessoire anormale entre les oreillettes et les ventricules, comme dans la maladie congénitale de Wolff, Parkinson et White, ou bien encore être causées par l'activité anormale du myocarde ischémique qui survit en marge de la cicatrice d'un infarctus. Ces tachycardies sont susceptibles de dégénérer en fibrillation ventriculaire, cause habituelle de la mort subite. L'électrocardiogramme (ECG) classique permet au médecin de reconnấtre une tachycardie et d'en faire une première analyse. Cependant, l'ECG ne permet pas une localisation suffisamment précise du myocarde arythmogène pour une étude approfondie du mécanisme de l'arythmie et pour diriger le traitement chirurgical de certaines tachycardies rebelles au traitement médical. Cet article traite de l'enregistrement invasif d'électrogrammes épicardiques et intracardiaques pour déterminer surtout l'origine des tachycardies ventriculaires.

\section{La cartographie de l'activation cardiaque}

La méthode traditionnelle de cartographie épicardique utilise des électrogrammes unipolaires ou bipolaires enregistrés l'un à la suite de l'autre en déplaçant une électrode exploratrice sur le cœur exposé [1, 2]. Les signaux sont amplifiés, puis visualisés sur un écran d'oscilloscope ou sur papier. On mesure manuellement les intervalles entre l'activation d'un électrogramme de référence et celle de chacun des points d'enregistrement. Enfin, on représente la séquence d'activation sur une carte constituée de lignes isochrones tracées à la main. On
R. Cardinal, P. Pagé, M. Shenasa, G. Fau gère, P. Savard : centre de recherche, hôpital Montréal, P.Q., Canada H4J 1 C5. 


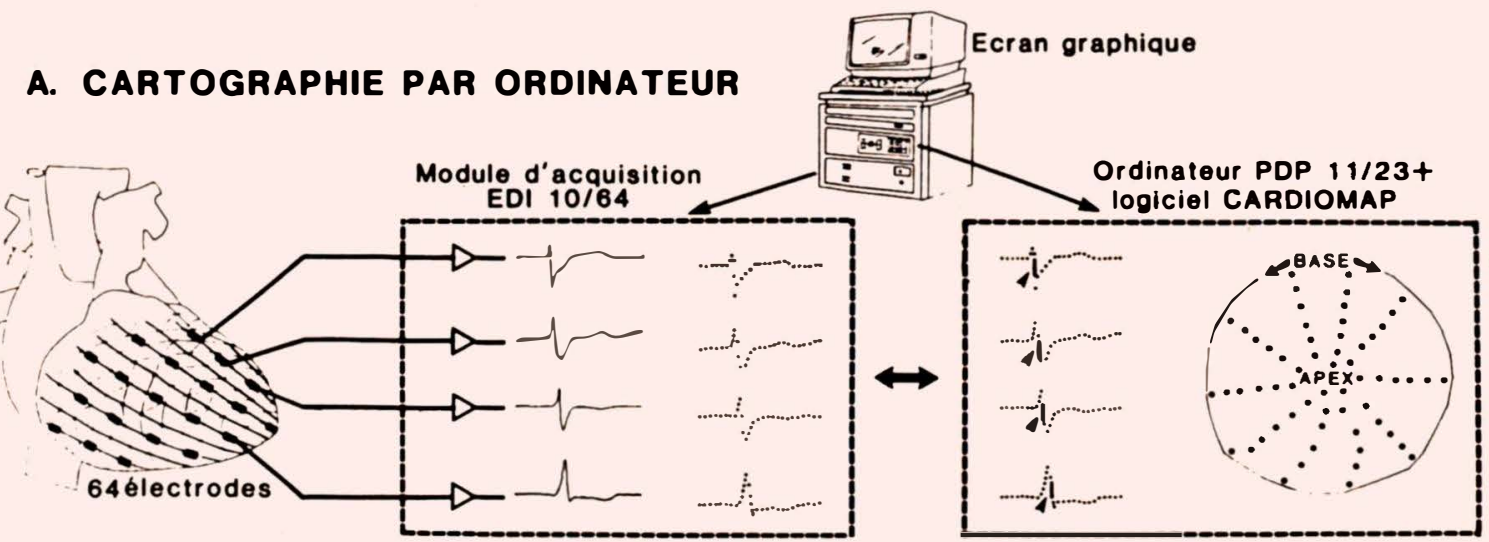

B. CARTOGRAPHIE EPICARDIQUE ON RYTHME SINUSAL
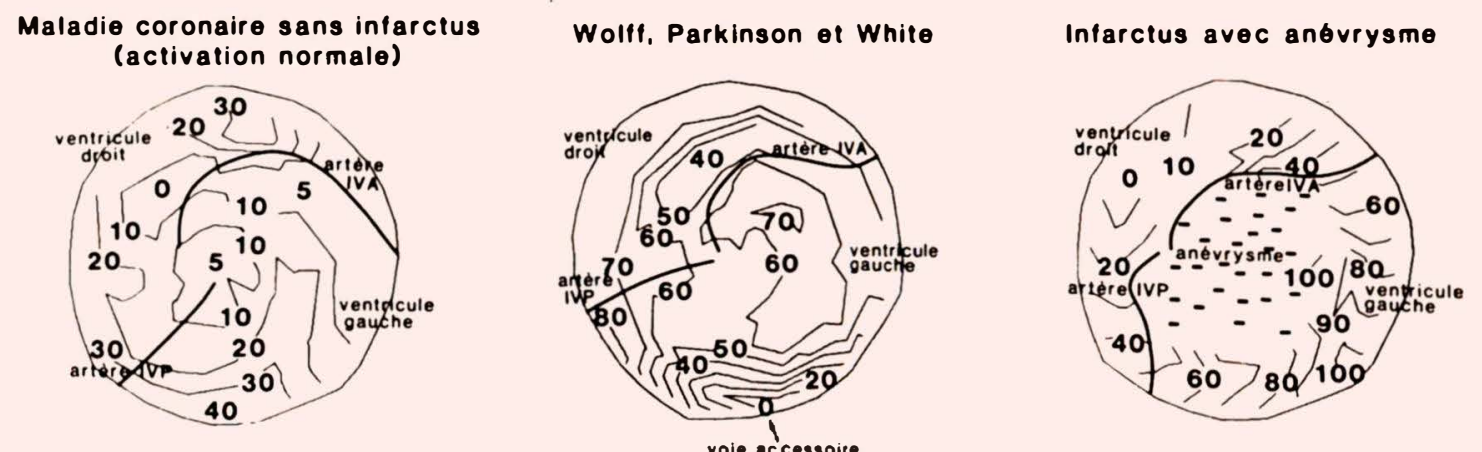

Figure 1. Cartographie de l'activation cardiaque. A. Schéma de l'acquisition et du traitement des électrogrammes pour déterminer la séquence de l'activation cardiaque selon la méthode récente de cartographie par ordinateur. B. Cartographie ventriculaire épicardique pendant le rythme sinusal dans trois maladies, dont la première (à gauche) n'affecte pas le processus normal d'activation. IVA = interventriculaire antérieure; IVP = interventriculaire postérieure.

l'obtient en combinant des mesures faites sur un grand nombre de battements successifs.

En rythme sinusal, cette technique d'enregistrement a été utilisée chez l'homme pour localiser les voies accessoires associées au syndrome de Wolff, Parkinson et White [3, 4].

Elle peut également localiser les potentiels tardifs associés à certaines formes de tachycardie ventriculaire rebelle [5, 6].

$\mathrm{La}$ détection de ces indicateurs électrophysiologiques du myocarde arythmogène, témoins d'une conduction lente, peut être utile dans $m / s n^{\circ} 6$ vol. 3 , juin 87 certains cas où la tachycardie par réentrée n'est pas reproductible en salle d'opération. La cartographie en rythme sinusal a été utilisée chez l'animal pour étudier l'action dépressive des antiarythmiques de la classe 1 sur la conduction normale [7] ou pathologique [8].

L'utilisation de l'ordinateur par Fontaine et al. [4,5] pour tracer les isochrones est un moment important dans l'évolution de la cartographie cardiaque puisque ces auteurs ont ainsi démontré l'intérêt d'une cartographie complète obtenue systématiquement et ont ouvert la possibilité d'accélérer le traitement des données. Malgré l'utilisation du compteur électronique pour mesurer les intervalles d'activation et l'automatisation du traçage des isochrones, la cartographie demeurait limitée par la durée prolongée de l'exploration point par point.

Grâce aux progrès récents de la technologie électronique et informatique, plusieurs groupes de chercheurs ont mis au point des systèmes qui leur permettent d'enregistrer simultanément tous les électrogrammes nécessaires [9-11]. La figure $1 A$ présente un schéma de notre système, qui 
5. Fontaine G, Guiraudon G, Frank R, et al. La cartographie épicardique et le traitement chirurgical par simple ventriculotomie de certaines tachycardies ventriculaires rebelles par réentrée. Arch Mal Cour 1975 ; 68 : 113-24.

6. Klein H, Karp RB, Kouchoukos NT Zorn GL, James TN, Waldo AL. Intraoperative electrophysiologic mapping of the ventricles during sinus rhythm in patients with a previous myocardial infarction. Circulation $1982 ; 66: 847-53$

7. Boissier JR, Giudicelli JF, Richer C, Bertin MC. Cartographie de dépolarisation ventriculaire épicardique et conduction intracardiaque. J Pharmacol 1975 ; 6 : 453-66.

8. Cardinal R, Janse MJ, van Eeden I, Werner $G$, Naumann d'Alnoncourt $C$, Durrer D. The effects of lidocaine on intracellular and extracellular potentials, activation and ventricular arrhythmias during acute regional ischemia in the isolated porcine heart. Circ Res $1981 ; 49: 792-806$

9. Ideker RE, Smith WM, Wallace AG, et al A computerized method for the rapid display of ventricular activation during the intraoperative study of arrhythmias. Circulation 1979 ; 59 : 449-58

10. De Bakker JMT, Janse MJ, van Capelle FJL, Durrer D. An interactive computer system for guiding the surgical treatment of life-threatening ventricular tachycardias. IEEE Trans Biomed Eng 1984; BME-31 : $362-8$.

11. Downar E, Parson ID, Mickleborough LL, Cameron DA, Yao LC, Waxman MB. On-line epicardial mapping of intraoperative ventricular arrhythmias : Initial clinical experience. $J A m$ Coll Cardiol 1984; 4 : 703-14.

12. Bonneau G, Tremblay G, Savard P, et al. A real-time cardiac mapping system : a description. IEEE Trans Biomed Eng 1987 (sous presse).

13. Escande D, Corabœuf E. Electrophysiologie cellulaire des foyers d'automatisme anormaux. Arch Mal Caur 1985; 78 : 1969-77.

14. Cardinal R, Savard P, Armour JA Nadeau RA, Carson DL, LeBlanc AR. Isochronal and isopotential mapping of ventricular tachycardia induced by electrical stimulation of local cardiac nerves in dogs. Can J Physiol Pharmacol 1986 ; 64 : 411-8.

15. Mehra R, Zeiler RH, Gough WB, ElSherif N. Reentrant ventricular arrhythmias in the late myocardial infarction period. 9. Electrophysiologic-anatomic correlation of reentrant circuits. Circulation 1983 ; 67 : 11-24.

16. Cardinal R, Savard P, Carson DL, Perry JB, Pagé P. Mapping of ventricular tachycardia induced by programmed stimulation in canine preparations of myocardial infarction. Circulation $1984 ; 70: 136-48$. comporte 64 voies d'enregistrement simultané [12]. Les contacts unipolaires ou bipolaires sont fixés à l'intérieur d'un bas extensible que le chirurgien enfile sur l'épicarde (à gauche). Dans cette configuration, les contacts sont uniformément disposés sur l'ensemble de la surface ventriculaire. Les électrodes sont branchées sur le module d'acquisition EDI 10/64 (Electrophysiologic Data Interface) mis au point par l'Institut de génie biomédical de Montréal. Ce module a une triple fonction : (a) l'isolement électrique du patient par un procédé électrooptique, (b) l'amplification des électrogrammes, et (c) leur conversion de la forme analogique à la forme numérique par un échantillonnage à des intervalles de deux millisecondes (ms), ou plus rapidement. Les signaux numérisés sont instantanément transférés à un ordinateur commercial (PDP11/23 +, Digital Equipment Corporation, Maynard, Massachusetts, USA) et stockés sur bande magnétique ou sur disque. Le module d'acquisition fonctionne sous le contrôle de l'ordinateur programmé avec notre logiciel Cardiomap.

L'opérateur communique avec ce logiciel au moyen d'un écran graphique et d'un clavier. Il démarre l'acquisition et en fixe les paramètres, comme le gain des amplificateurs, et peut l'interrompre à tout moment pour choisir des cycles d'activation cardiaque et en faire la cartographie. La détection des points de dépolarisation sur les électrogrammes et le traçage des cartes isochrones sont automatiques. Cependant, l'opérateur peut intervenir dans la détection au cours de la révision des 64 électrogrammes qui lui sont présentés un à un sur l'écran. Les cartes isochrones des cycles d'activation choisis sont disponibles en quelques minutes.

La cartographie épicardique réalisée lors d'une intervention de pontage aorto-coronaire chez un malade n'ayant jamais présenté d'infarctus du myocarde nous permet de retrouver les caractéristiques générales de l'activation ven- triculaire normale en rythme sinusal (figure $1 B$, à gauche). Dans la représentation polaire de la surface épicardique, la base des ventricules est située le long de la circonférence du cercle et leur apex au centre. L'activation épicardique débute sur la paroi antérieure du ventricule droit. Quelques $5 \mathrm{~ms}$ plus tard, l'activation survient dans la région paraseptale antérieure du ventricule gauche, ainsi que dans sa région postéroapicale. Les autres régions de l'épicarde sont ensuite activées. L'activation la plus tardive se situe dans les régions postérieures et latérales de la base du ventricule gauche (40 ms).

Par ailleurs, chez un patient qui présente le syndrome de Wolff, Parkinson et White (figure $1 B$, au centre), le début de la séquence d'activation ventriculaire est détecté près du sillon auriculoventriculaire sur la paroi latérale gauche, là où s'insère la voie accessoire. L'activation de l'épicarde ventriculaire droit, normalement la plus précoce, survient $40 \mathrm{~ms}$ plus tard.

Chez un patient porteur d'un anévrysme du ventricule gauche développé à la suite d'un infarctus antéroseptal (figure $1 B$, à droite), l'activation première en rythme sinusal survient de façon normale à l'épicarde ventriculaire droit. La région centrale de la carte ne présente pas d'électrogramme en raison de la fibrose de la paroi anévrysmale. Dans cet exemple, le temps d'activation épicardique total est de $106 \mathrm{~ms}$, intervalle considérablement plus long que la normale.

\section{Études expérimentales}

Traditionnellement, on classe les mécanismes arythmogènes selon deux types : a) l'automatisme accéléré ou anormal, dont l'activité déclenchée [13], et b) les troubles de la conduction et de l'excitabilité qui causent la réentrée. A cause de la multiplicité des facteurs électrophysiologiques, métaboliques et nerveux perturbés, le myocarde arythmogène n'est pas une entité pathologique 
simple dont on pourrait décrire les caractéristiques de façon univoque. C'est pourquoi il est nécessaire d'étudier les conditions arythmogènes chez l'animal lors de plusieurs situations expérimentales. La méthode cartographique s'est avérée très utile pour étudier ces mécanismes pendant les tachycardies ventriculaires provoquées par la stimulation anormale du système nerveux autonome [14] ou par l'occlusion coronarienne. A la suite de l'occlusion d'une artère coronaire, plusieurs phases d'arythmies ventriculaires se succèdent. Elles sont dues aux modifications de l'activité électrique d'une couche de cellules ischémiques qui survivent en marge du tissu nécrosé (figure $2 A$ : la surface ventriculaire déployée est vue de face, sur un plan épicardique et un plan endocardique). Pendant le rythme ventriculaire dû à l'automatisme accéléré des fibres de Purkinje (figure $2 B$ ), on voit l'activité électrique se propager à partir d'un foyer endocardique. Les tachycardies ventriculaires par réentrée (figure $2 C$ ) peuvent survenir spontanément ou être provoquées par la stimulation rapide des ventricules (S1, S2, S3, S4). La stimulation programmée favorise l'apparition d'un bloc de conduction dans le tissu ischémique qui devient réfractaire. L'onde contourne ensuite la zone du bloc et s'y propagera ultérieurement en conduction rétrograde pour initier la réentrée. La taille du circuit définit alors une macro- ou une microréentrée. Chez le chien, ces tachycardies peuvent être attribuées à un double circuit de réentrée dans le myocarde sousépicardique $[15,16]$. La carte isochrone prend alors (figure $2 C$ ) une forme de huit dont l'isthme est situé au centre de la région ischémique et constitue la voie de conduction lente nécessaire pour que le myocarde sain recouvre son excitabilité d'un cycle à l'autre. Le point d'origine de la tachycardie est celui où l'activation électrique est la plus précoce. Celle-ci survient dans la partie moyenne ou tardive de la diastole, avant le début du complexe QRS

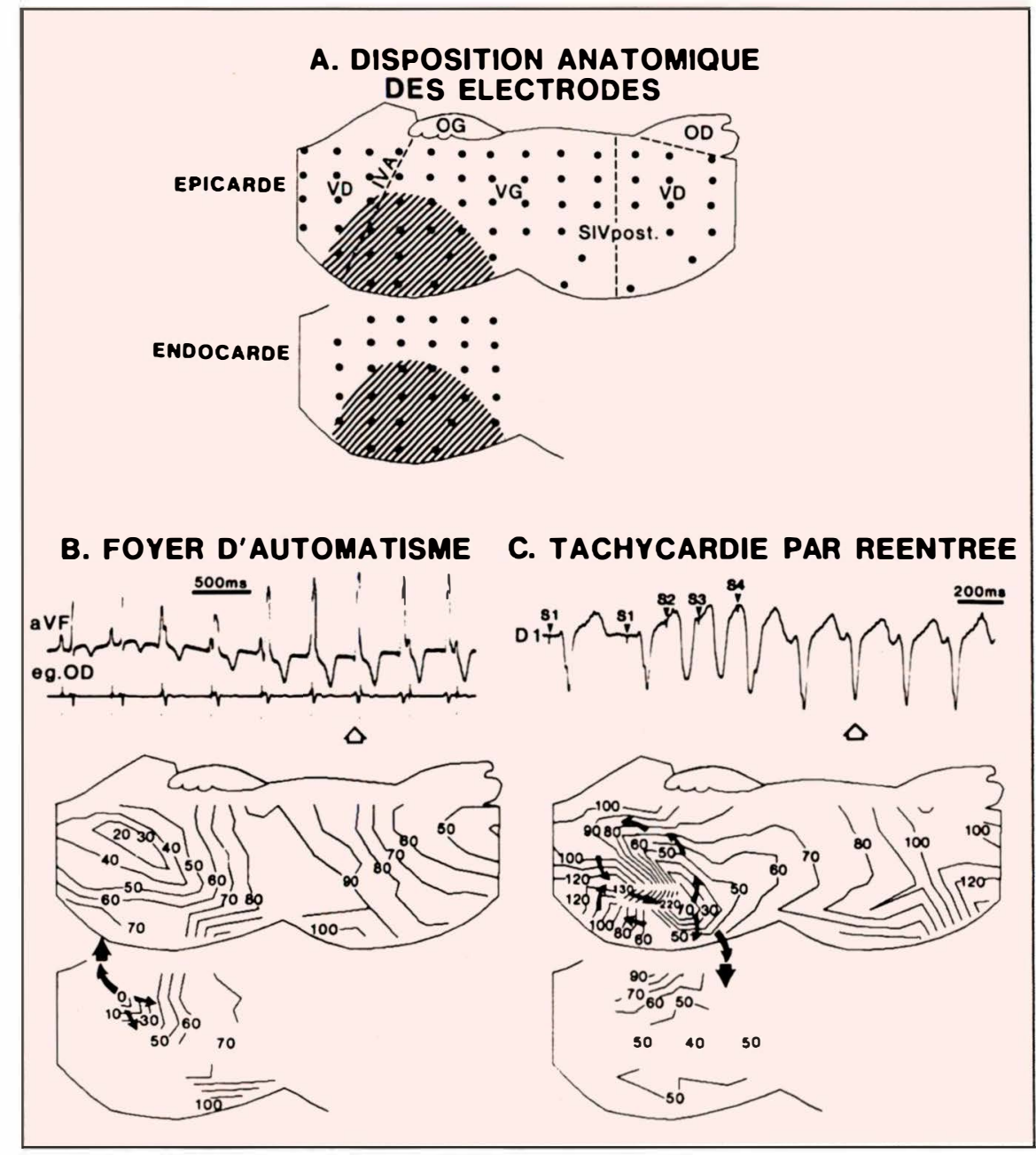

Figure 2. Séquence d'activation d'une tachycardie ventriculaire causée par un foyer d'automatisme accéléré situé à l'endocarde (B), et d'une tachycardie ventriculaire par réentrée à l'épicarde (C). Sur le schéma des ventricules (A) sont indiquées la disposition des électrodes et la position du myocarde ischémique (région hachurée) trois jours après l'occlusion de l'artère interventriculaire antérieure (IVA) chez le chien. OD, VD = oreillette droite, ventricule droit ; $O G, V G=$ oreillette gauche, ventricule gauche; SIV post. = sillon interventriculaire postérieur.

de l'ECG de surface. Dans notre exemple, cette région est située là où la voie de conduction lente se termine dans le myocarde sain, et son activation se produit au moment où commence le cycle d'activation suivant (220/0). Les médicaments antiarythmiques peuvent agir sur le myocarde ischémique pour inactiver cette zone de conduction lente [8], ou sur le myocarde sain en prolongeant suffisamment sa période réfractaire pour que l'onde qui émerge du myocarde ischémique ne soit plus en mesure d'en déclencher la réactivation [17]. Pour le traitement chirurgical des tachycardies rebelles chez l'homme, on doit localiser la région d'origine de la tachycardie et en faire l'ablation.

\section{Cartographie} endocavitaire en clinique

C'est en 1972 que Wellens, Schuilenberg et Durrer [18] ont pu, par la stimulation programmée, induire et interrompre des tachycardies ventriculaires chez cinq malades en phase chronique d'un infarctus. Ces études de provocation sont maintenant pratiquées chez le malade conscient dans le but de choisir un médi- 


\section{RÉFÉRENCES}

17. Cardinal R, Sasyniuk BI. Electrophysiological effects of bretylium tosylate on subendocardial Purkinje fibers from infarcted canine hearts. J Pharmacol Exp Ther 1978; 204 : 159-74.

18. Wellens HJJ, Schuilenberg RM, Durrer D. Electrical stimulation of the heart in patients with ventricular tachycardia. Circulation $1972 ; 46$ : 216-26

19. Fisher JD, Cohen HL, Mehra R, Altschuler $\mathrm{H}$, Escher DJW, Furman S. Cardiac pacing and pacemakers. II. Serial electrophysiologicpharmacologic testing for control of recurrent tachyarrhythmias. Am Heart J 1977 ; 93 : 658-68.

20. Lévy $S$, Cointe $R$, Metge $M$, Faugère $G$, Valeix B, Gérard R. Bepridil for recurrent sustained ventricular tachycardias: Assessment using electrophysiologic testing. Am J Cardiol $1984 ; 54: 579-81$.

21. Touboul P, Atallah G, Kirkorian G, et al. Electrophysiologic effects of cibenzoline in humans related to dose and plasma concentration. Am Heart J 1986 ; 112 : 333-9.

22. Shenasa $M$, Fromer $M$, Faugère $G$, et al. Safety and efficacy of intravenous and oral diltiazem in the Wolff-Parkinson-White syndrome. Am J Cardiol 1.987; 59 : 301-6.

23. Josephson ME, Horowitz LN, Farshidi A, Spear JF, Kastor JA, Moore EN. Recurrent sustained ventricular tachycardia. II. Endocardial mapping. Circulation 1978; 57 : 440-7.

24. De Bakker JMT, Janse MJ, van Capelle FJL, Durrer D. Endocardial mapping by simultaneous recording of endocardial electrograms during cardiac surgery for ventricular aneurysm. J Am Coll Cardiol $1983 ; 2$ : 947-53.

25. Downar E, Mickleborough LL, Harris L, Parson ID. Mapping of endocardial activation during ventricular tachycardia. A " closed. heart " procedure. J Am Coll Cardiol $1986 ; 7$ : 234A.

26. Josephson ME, Wit AL. Fractionated electrical activity and continuous electrical activity: fact or artifact. Circulation $1984 ; 70: 529-32$.

27. Miller JM, Harken AH, Hargrove WC, Josephson ME. Pattern of endocardial activation during sustained ventricular tachycardia. J Am Coll Cardiol 1985; 6 : 1280-7.

28. Fontaine G, Guiraudon G, Frank R, Fillette F, Cabrol C, Grosgogeat Y. Surgical management of ventricular tachycardia unrelated to myocardial ischemia or infarction. $A m$ $J$ Cardiol 1982 ; 49 : 397-410.

29. Faugère $G$, Savard $P$, Nadeau $R$, Derome D, Shenasa M, Pagé P, Guardo R. Characterization of the spatial distribution of late ventricular potentials by body surface mapping in patients with ventricular tachycardia. cament antiarythmique ou un mode de stimulation par pacemaker efficace [19-22]. A défaut, la cartographie endocardique est pratiquée en prévision du traitement par la chirurgie ou par les méthodes ablatives endocavitaires (électrofulguration). Une sondeélectrode flexible est introduite dans le ventricule droit par ponction veineuse percutanée ou dans le ventricule gauche par ponction artérielle et déplacée sous contrôle cinéfluoroscopique. On peut ainsi enregistrer les électrogrammes d'une vingtaine de sites endocardiques. Ce type de cartographie pose cependant les problèmes suivants : la résolution spatiale est faible ; la transposition de l'information d'une vue fluoroscopique bidimensionnelle à la représentation tridimensionnelle utilisée par le chirurgien est difficile ; les électrogrammes n'étant pas recueillis simultanément, il est nécessaire d'induire la tachycardie à plusieurs reprises au risque d'en changer la morphologie ; et enfin, la procédure sera incomplète ou impraticable si la tachycardie est mal tolérée hémodynamiquement. Néanmoins, Josephson et al [23] ont démontré par cette méthode que l'origine des tachycardies ventriculaires est habituellement située en marge de l'anévirysme ou de la cicatrice de l'infarctus.

\section{Cartographie épicardique peropératoire}

L'origine de la tachycardie ventriculaire est finalement précisée en salle d'opération. Il est possible d'enregistrer successivement un grand nombre d'électrogrammes avec une électrode exploratrice. Cependant, il faut les recueillir au cours de la tachycardie, ce qui est souvent difficile car l'arythmie peut être d'une durée trop courte ou d'une morphologie trop variable pour qu'on puisse dresser une carte isochrone complète. L'induction de quelques complexes ventriculaires n'est pas nécessairement représentative de la tachycardie ventriculaire clinique. L'anesthésie générale, l'hypothermie myocardique et les modifications de la tension intramurale du ventricule gauche peuvent rendre difficile l'induction de la tachycardie et en raccourcir la durée. L'enregistrement simultané au moyen d'un système informatisé nous permet d'obtenir en un seul moment toute l'information pertinente. Chez le patient porteur d'un anévrysme du ventricule gauche dont la carte en rythme sinusal a été présentée dans la figure $1 B$, on a provoqué et cartographié des tachycardies avec deux morphologies distinctes. Dans l'une (figure $3 A$ ), l'activation épicardique la plus précoce a été détectée sur le bord gauche de l'anévrysme, et dans l'autre sur le bord droit (figure $3 B$ ). Cependant, la durée de l'activation épicardique (105 ou $150 \mathrm{~ms}$ ) est bien inférieure au cycle de la tachycardie (320 ou $280 \mathrm{~ms}$ ) et ne peut pas rendre compte d'un mécanisme de réentrée utilisant un grand circuit ventriculaire. De plus, on peut se demander si ces deux morphologies sont liées à l'expression de deux points d'origine distincts ou à deux manifestations d'une même origine endocardique.

\section{Cartographie endocardique peropératoire}

Chez l'homme, l'origine de la tachycardie ventriculaire est le plus souvent détectée à l'endocarde [2, 23]. En présence d'un anévrysme du ventricule gauche, une incision de l'anévrysme par laquelle on passe une électrode exploratrice permet d'enregistrer successivement plusieurs électrogrammes endocardiques pendant la tachycardie. Certains en font l'enregistrement simultané en insérant à l'intérieur du ventricule un ballon porteur de plusieurs électrodes [24, 25]. Dans la figure 4, l'intérieur du ventricule gauche est représenté par un cercle dont le centre est occupé par les orifices valvulaires gauches, et dont la périphérie correspond à la marge de résection de l'anévrysme. Pendant la tachycardie, la carte d'activation peut présenter une séquence circulaire continue dans le sens antihoraire, 


\section{CARTOGRAPHIE EPICARDIQUE pendant la TACHYCARDIE VENTRICULAIRE}

\section{A.Morphologie de bloc de branche droite}

B.Morphologie de bloc de branche gauche
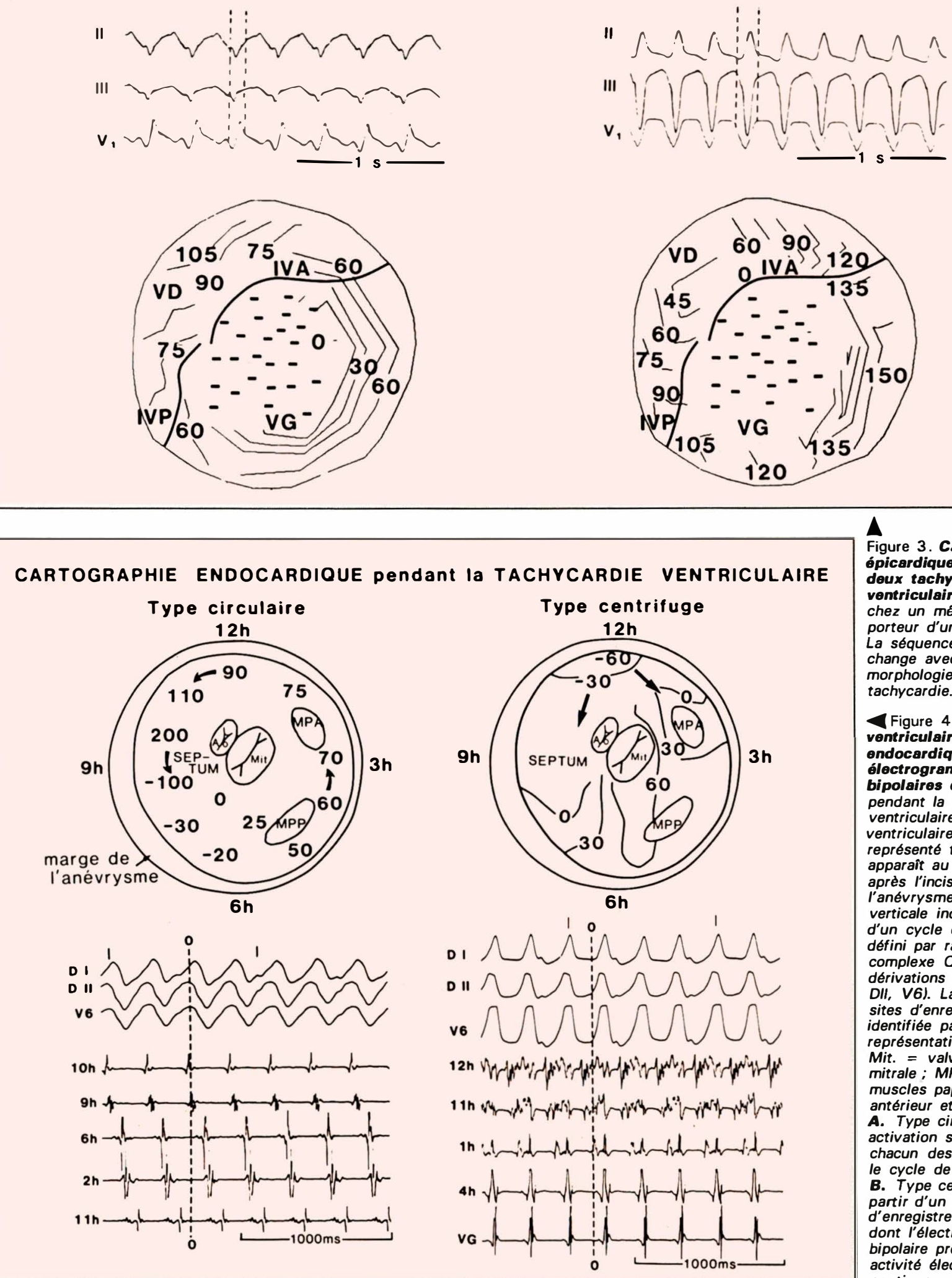

Figure 3. Cartes

épicardiques pendent deux tochycordios ventriculaires provoquées chez un même patient porteur d'un anévrysme. La séquence d'activation change avec la morphologie de la tachycardie.

$\checkmark$ Figure 4. Cartographie ventriculaire

ondocardique ot électrogrammos bipolairos enregistrés pendant la tachycardie ventriculaire. L'endocarde ventriculaire gauche est représenté tel qu'il

apparaît au chirurgien après l'incision de l'anévrysme. La ligne verticale indique le début d'un cycle d'activation défini par rapport au

complexe ORS des

dérivations de surface (DI DII, V6). La position des sites d'enregistrement est identifié par une représentation horaire. Ao, Mit. $=$ valves aortique et mitrale ; MPA, MPP =

muscles papillaires

muscles papillaires

antérieur et postérieur. A. Type circulaire:
activation successive de chacun des sites pendant le cycle de la tachycardie.

B. Type centrifuge à partir d'un point

partir d'un point

dont l'électrogramme

bipolaire présente une activité électrique continue. 
qui suggère une macroréentrée endocardique (figure $4 A$ ). On peut suivre sur les électrogrammes cette progression régulière de l'activité endocardique. Cependant, il n'est pas exclu qu'il s'agisse plutôt de la dispersion circulaire du front d'onde à partir d'un foyer de microréentrée, voire d'activité automatique déclenchée, qui présenterait un bloc de conduction dans une direction. Le mouvement circulaire ressemble parfois à une figure en forme de huit telle celle décrite dans la figure 2.

Dans la figure $4 B$, obtenue chez un autre patient porteur d'un anévrysme antérieur, la carte endocardique montre que l'activité la plus précoce se situe en un seul point sur la paroi antérieure du ventricule gauche et que la dispersion de l'onde à partir de ce point est centrifuge plutôt que circulaire. L'électrogramme bipolaire enregistré au point d'origine de la tachycardie présente une activité électrique presque continue. Comme le signal est organisé et qu'il se prolonge d'un cycle de la tachycardie au suivant, il est séduisant de penser que ce type d'activité représente une microréentrée. Plusieurs auteurs [26] soutiennent que la présence de l'activité électrique continue est essentielle au maintien de la tachycardie.

Miller et al. [27] ont retrouvé à l'endocarde le type d'activation centrifuge dans 90 tachycardies et le type circulaire continu dans 11 des 122 tachycardies provoquées chez 55 patients, l'activation n'ayant pu être décrite pendant 21 tachycardies en raison du nombre insuffisant d'électrogrammes. Toutefois, il ne faudrait pas négliger la participation éventuelle du myocarde intramural dans le mécanisme de la tachycardie. L'implantation d'électrodes plongeantes à sites d'enregistrement étagés nous permet d'étudier le rôle des couches intramurales, et de comparer l'activation sousépicardique et sous-endocardique. La résection de l'endocarde dans la région d'origine de la tachycardie est la technique chirurgicale la traiter les tachycardies ventriculaires liées à la maladie coronaire. Les résultats de ces opérations sont remarquables puisqu'elles assurent le contrôle des tachycardies ventriculaires rebelles dans une proportion de 65 à $90 \%$, selon les séries et les modes d'évaluation, mais avec un risque opératoire non négligeable. Par ailleurs, le traitement chirurgical du syndrome de Wolff, Parkinson et White dirigé par la cartographie permet un taux de succès encore supérieur. Mentionnons que la cartographie est utilisée également pour diriger le traitement chirurgical des tachycardies ventriculaires qui surviennent dans le cœur apparemment normal ou myocardiopathique, en l'absence de la maladie coronaire [28].

Dans leur état actuel, ces méthodes de cartographie ne nous permettent de localiser les zones arythmogènes qu'avec une précision de l'ordre de quelques $\mathrm{cm}^{2}$. Ceci n'est pas suffisant pour déterminer avec certitude le mécanisme des tachycardies ventriculaires. Bien qu'on attribue généralement à la réentrée celles qui sont inductibles par la stimulation programmée, pouvons-nous exclure complètement, dans ces conditions, la possibilité d'une activité automatique déclenchée par une stimulation initiatrice [13] ?

Une meilleure connaissance des mécanismes arythmogènes et une précision accrue de nos méthodes permettront de meilleurs résultats chirurgicaux, et notamment de réduire la quantité de myocarde réséqué (ou inactivé par la cryochirurgie) afin de préserver la fonction ventriculaire. L'enregistrement non invasif des potentiels thoraciques [29] pourrait également contribuer à la localisation des zones arythmogènes qui passe actuellement par des méthodes sanglantes

\section{Remerciements}

Les auteurs remercient les professeurs Réginald Nadeau, Robert Cossette, Fernand Roberge, Robert Guardo et Robert Leblanc, sans qui ce travail n'aurait pu être réalisé.

\section{Summary}

Recent advances in electronics and computer technology enables us to simultaneously record 64 cardiac electrograms, and to construct epicardial and endocardial activation maps during ventricular tachycardia. In the late phase following myocardial infarction, ventricular tachycardia can be induced by programmed stimulation in patients with spontaneously recurring episodes of tachycardia. Experimental studies suggest that inducible tachycardia may be caused by reentry in ischemic myocardium surviving near by the fibrotic scar. The earliest epicardial breakthrough determined by intraoperative mapping in patients occur at the border of their scar ; however, the total duration of epicardial activation is much shorter than the tachycardia cycle length, and the epicardial map does not display a macro reentry pattern. After incision of the scar or aneurysm, two patterns of endocardial activation are found : 1) a circular pattern consistent with a macro-reentry mechanism ; or 2) centrifugal spread from a single or multiple sites (microreentry or triggered activity ?). Endocardial resection guided by mapping is the most widely used approach for antiarhythmic surgery, which achieves arrhythmia control in $65 \%$ to $90 \%$ of patients. A better understanding of the mechanism of ventricular tachycardia and greater accuracy in the localization of its origin may increase the efficacy of antiarrhythmic surgery.

\section{TIRÉS A PART}

R. Cardinal : centre de recherche, hôpital du Sacré-Cœur, 5400 boulevard Gouin ouest, Montréal, P.Q., Canada H4J 1 C5. 